\title{
A New Conservative Allen-Cahn Type Ohta-Kawaski Phase-Field Model for Diblock Copolymers and Its Numerical Approximations
}

\author{
Shuang Geng ${ }^{1}$, Tongmao $\mathrm{Li}^{1}$, Qiongwei $\mathrm{Ye}^{2, *}$ and Xiaofeng Yang ${ }^{3, *}$ \\ ${ }^{1}$ College of Management, Shenzhen University, Shenzhen, Guangdong 518060, China \\ 2 Business School, Yunnan University of Finance and Economics, Kunming, \\ Yunnan 650221, China \\ ${ }^{3}$ Department of Mathematics, University of South Carolina, Columbia, SC 29208, USA
}

Received 15 September 2020; Accepted (in revised version) 8 January 2021

\begin{abstract}
We develop a new conservative Allen-Cahn phase-field model for diblock copolymers in this paper by using the Allen-Cahn type gradient flow approach for the classical Ohta-Kawaski free energy. The change in volume fraction of two composing monomers is eliminated by using a nonlocal Lagrange multiplier. Based on the recently developed stabilized Scalar Auxiliary Variable method, we have further developed an effective numerical scheme to solve the model. The scheme is highly efficient and only two linear and decoupled equations are needed to solve at every time step. We then prove that the numerical method is unconditionally energy stable, the stability and accuracy of the new scheme are demonstrated by numerous numerical examples conducted. By qualitatively comparing the equilibrium solution obtained by the new model and the classic Cahn-Hilliard model, we illustrate the effectiveness of the new model.
\end{abstract}

AMS subject classifications: 65N12, 65P40, 65Z05

Key words: Phase-field, Diblock copolymer, Allen-Cahn, nonlocal, second order, unconditional energy stability.

\section{Introduction}

The molecules of a block copolymer are composed of two or more segments of simple polymers (blocks). For instance, polymers of AB-type (called diblock) mean that every single chain is formed by a segment composed of the monomer $\mathrm{A}$ and a segment with monomer B. Recently, block copolymers have attracted great interest in the field

${ }^{*}$ Corresponding author.

Emails: gs@szu.edu.cn (S. Geng), litongmao@email.szu.edu.cn (T. Li), yqw@ynufe.edu.cn (Q. Ye), xfyang@ math.sc.edu (X. Yang) 
of science and engineering due to their highly adjustable self-assembly property in the nanoscale. That is, the copolymer system can self-assemble into various nanostructures, such as lamellar, cylinders, gyroids, and BCC (body-centered-cubic) phases, etc. To simulate the copolymer patterns and understand the process of formation of the copolymer chain, the diffusive interface phase-field method had been widely used as the effective modeling and simulation tool for this particular subject.

The basic idea of the phase-field method is to introduce a labeling function (phasefield variable) to represent the difference between the local volume fractions of the two constituent monomers, cf. $[3,5,11,16,17,22,23]$. For the diblock copolymer model, the total free energy usually consists of two parts, one of which is the linear part of gradient entropy, and the other is the nonlinear part containing the double-well potential and the so-called Ohta-Kawasaki nonlocal potential, see the pioneering work by Ohta and Kawaski in [17]. By using the variational method to minimize the assumed total free energy in the assumed metric, the governing PDE model can be further derived.

We note that the phase-field variable used in the diblock copolymer model is interpreted as the local volume fraction of the two monomers, so it is expected to conserve over time. Therefore, the model is generally derived using the Cahn-Hilliard dynamics $\left(\mathrm{H}^{-1}\right.$-gradient flow approach), thereby obtaining a fourth-order system. It is well-known that there is another gradient flow approach in phase-field modeling, the so-called AllenCahn dynamics ( $\mathrm{L}^{2}$-gradient flow approach). Note that the PDE system generated by the Allen-Cahn type model is second-order less than the PDE system of the Cahn-Hilliard model, which in turn brings weaker CFL conditions for grid size in time and/or space. Therefore, from a numerical point of view, the second-order Allen-Cahn model is preferable to the fourth-order Cahn-Hilliard model.

However, the Allen-Cahn model has an inherent defect that the volume cannot usually be conserved. To fix this issue, Rubinstein and Sternberg developed a so-called conservative Allen-Cahn equation, in which the variance of the total volume is offset by using a nonlocal Lagrange multiplier while retaining the law of energy dissipation. Inspired by this ingenious nonlocal technique, the first goal of this paper is to rebuild the diblock copolymer model using the lower-order Allen-Cahn dynamics. More precisely, after applying the variational method to the classic Ohta-Kawaski free energy in the $L^{2}$ space and adding a nonlocal Lagrange multiplier to offset the volume change, we arrive at a conservative nonlocal Allen-Cahn type PDE model for diblock copolymers. However, when designing a reliable and effective numerical algorithm for solving the new model, some new difficulties are encountered, especially how to discretize the newly added nonlocal term. In other words, if we directly apply the known methods that can successfully solve the local phase-field model, including simple-implicit [9], explicit [12, 13, 20, 27], convex-splitting $[1,8]$, etc., to deal with the newly obtained nonlocal model, the resulting numerical scheme will inevitably fail to maintain unconditional energy stability, or become a nonlinear scheme with actual high computational cost $[15,27]$.

Therefore, the second purpose of this article is to construct an effective numerical scheme to solve the new conservative Allen-Cahn diblock copolymer model. To this end, 
we modify the recently developed Scalar Auxiliary Variable (SAV) approach to be the socalled "stabilized-SAV" approach [21,24-26,28,29], which adds a key linear stabilization term to improve stability. The detailed implementation method is to define a scalar auxiliary variable first and then reformulate the PDE model accordingly. By using an inverse linear operator, the nonlocal term eventually disappears, and we only need to solve two linear systems with constant coefficients at each time step. The scheme is then be proved to be unconditionally energy stable. The numerical results of the classical Cahn-Hilliard phase-field model are compared qualitatively to simulate a large number of numerical examples in 2D and 3D spaces, which proves the effectiveness of the new model. The stability and accuracy of the developed scheme are also demonstrated numerically.

The rest of the article is structured as follows. In Section 2, the governing equations of the new Allen-Cahn type system are developed and the corresponding energy law is derived. In Section 3, the temporal second-order numerical algorithm is developed to solve the proposed model. In Section 4, we give numerous numerical simulations to demonstrate the accuracy and stability. We present some conclusive remarks in Section 5 .

\section{Model equations}

Now we present the new conserved Allen-Cahn type phase-field model for diblock copolymers. Assuming a system consists of molecules of two monomers A and B. We introduce a scalar labeling function $\phi(x, t)$ to represent the local volume fraction of these two components where $x \in \Omega^{d}, d=2,3$ and time $t \in[0, T)$. Hence, by using the Allen-Cahn dynamics, i.e., the $L^{2}$-gradient flow, the state of the system that relates to the time change of $\phi$ is described by the following phenomenological mesoscopic dynamic equation

$$
\frac{1}{M} \phi_{t}+\frac{\delta E(\phi)}{\delta \phi}=0
$$

where $M$ is a positive constant that represents the mobility of relaxation dynamics, $E(\phi)$ is a coarse-grained free energy functional that is postulated as (cf. $[2,6,7,10]$ ),

$$
E(\phi)=\int_{\Omega}\left(\frac{\epsilon^{2}}{2}|\nabla \phi|^{2}+F(\phi)\right) d x+\frac{\alpha \epsilon^{2}}{2} \int_{\Omega} \int_{\Omega} G(x-y)(\phi(x)-\bar{\phi})(\phi(y)-\bar{\phi}) d x d y,
$$

where $\epsilon$ is the gradient energy coefficient, the function $F(\phi)=\left(\phi^{2}-1\right)^{2}$ is the nonlinear double-well potential that takes $\phi= \pm 1$ as two minima. The function $\bar{\phi}(t)$ is defined as

$$
\bar{\phi}(t):=\frac{1}{|\Omega|} \int_{\Omega} \phi(x, t) d x,
$$

$G$ is the Green's function that reads as

$$
G(x-y)=-\delta(x-y),
$$


with the periodic boundary condition, $\delta$ is a Dirac delta function, $\alpha$ is the nonlocal positive parameter to characterizes the nonlocal potential and the molecular chain length, and the last term in (2.2) is the so-called nonlocal Ohta-Kawasaki functional.

We define the inverse Laplace operator $\psi=(-\Delta)^{-1} \phi$ as

$$
\left\{\begin{array}{c}
-\Delta \psi=\phi \\
\int_{\Omega} \psi d x=0
\end{array}\right.
$$

with some specific boundary conditions, where

$$
\phi \in L_{0}^{2}(\Omega):=\left\{\phi \in L^{2}(\Omega): \int_{\Omega} \phi d x=0\right\} .
$$

By taking variational derivative of the total free energy described in (2.2), the Allen-Cahn PDE system (2.1) turns into

$$
\begin{aligned}
& \frac{1}{M} \phi_{t}-\epsilon^{2} \Delta \phi+f(\phi)+\alpha \epsilon^{2} \psi=0, \\
& \psi=(-\Delta)^{-1}(\phi-\bar{\phi}),
\end{aligned}
$$

where $f(\phi)=F^{\prime}(\phi)$.

It is obvious that the volume fraction $\int_{\Omega} \phi(x, t) d x$ of the above system (2.6a)-(2.6b) is not conservative. To fix this, a nonlocal type Lagrange multiplier is added into the system thus volume variance caused by the nonlinear term $f(\phi)$ is eliminated thoroughly [18]. The new conservative Allen-Cahn type system reads as

$$
\begin{aligned}
& \frac{1}{M} \phi_{t}-\epsilon^{2} \Delta \phi+f(\phi)+\alpha \epsilon^{2} \psi-\frac{1}{|\Omega|} \int_{\Omega} f(\phi) d x=0, \\
& \psi=(-\Delta)^{-1}(\phi-\bar{\phi}) .
\end{aligned}
$$

In this work, we will use the periodic boundary condition such that all complexities caused by the boundary integrals are removed. One can also choose another alternative boundary conditions, e.g., the no-flux type boundary conditions that read as

$$
\left.\partial_{\mathbf{n}} \phi\right|_{\partial \Omega}=\left.\partial_{\mathbf{n}} \psi\right|_{\partial \Omega}=0,
$$

here, $\mathbf{n}$ is the outward normal of $\Omega$. All numerical analyses derived in this paper are also applicable to no-flux boundary conditions.

It is easy to show that the new model $(2.7 \mathrm{a})-(2.7 \mathrm{~b})$ conserves the volume. More precisely, we take the $L^{2}$ inner product of (2.7a) with 1 to obtain the following volume conservation property that reads as

$$
\frac{d}{d t} \int_{\Omega} \phi(x, t) d x=0
$$


Furthermore, the new conserved system (2.7a)-(2.7b) follows the same energy dissipation law as the non-conserved system (2.6a)-(2.6b). First, by taking the $L^{2}$ inner product of (2.7a) with $\phi_{t}$, and applying integration by parts, we obtain

$$
\frac{d}{d t} \int_{\Omega}\left(\frac{\epsilon^{2}}{2}|\nabla \phi|^{2}+F(\phi)\right) d x+\alpha \epsilon^{2}\left(\psi, \phi_{t}\right)=-\frac{1}{M}\left\|\phi_{t}\right\|^{2},
$$

where we use the volume-conserved property (2.9) to handle the nonlocal term, i.e.,

$$
\left(\int_{\Omega} f(\phi) d x, \phi_{t}\right)=0 .
$$

Second, to obtain $\left(\psi, \phi_{t}\right)$, we rewrite $(2.6 \mathrm{~b})$ as the form (2.5), i.e.,

$$
-\Delta \psi=\phi-\bar{\phi} \quad \text { with } \int_{\Omega} \psi d x=0
$$

and then take the time derivative for the above equation to get

$$
-\Delta \psi_{t}=\phi_{t}-\bar{\phi}_{t} .
$$

Taking the $L^{2}$ inner product of the above equation with $\alpha \epsilon^{2} \psi$, we find

$$
\frac{d}{d t} \int_{\Omega} \frac{\alpha \epsilon^{2}}{2}|\nabla \psi|^{2} d x=\alpha \epsilon^{2}\left(\psi, \phi_{t}\right)
$$

where $\left(\bar{\phi}_{t}, \psi\right)=0$ due to the zero-mean property of $\psi$. By combining (2.10) and (2.12), we obtain the energy decaying law that reads as

$$
\frac{d}{d t} E(\phi, \psi)=-\frac{1}{M}\left\|\phi_{t}\right\|^{2},
$$

where

$$
E(\phi, \psi)=\int_{\Omega}\left(\frac{\epsilon^{2}}{2}|\nabla \phi|^{2}+F(\phi)+\frac{\alpha \epsilon^{2}}{2}|\nabla \psi|^{2}\right) d x .
$$

Remark 2.1. By using the $H^{-1}$ gradient flow approach, the Cahn-Hilliard type phasefield model of diblock copolymers reads as (cf. [3,5,11,16,17,22, 23]),

$$
\begin{aligned}
& \phi_{t}=M \Delta w, \\
& w=-\epsilon^{2} \Delta \phi+f(\phi)+\alpha \epsilon^{2} \psi, \\
& \psi=(-\Delta)^{-1}(\phi-\bar{\phi}),
\end{aligned}
$$

where $w$ is the chemical potential; or an equivalent form as

$$
\begin{aligned}
& \phi_{t}=M\left(\Delta \mu-\alpha \epsilon^{2}(\phi-\bar{\phi})\right), \\
& \mu=-\epsilon^{2} \Delta \phi+f(\phi) .
\end{aligned}
$$

The energy dissipation law followed by the Cahn-Hilliard model reads as

$$
\frac{d}{d t} E(\phi, \psi)=-M\|\nabla w\|^{2}
$$




\section{Numerical schemes}

Now we develop a temporal second-order time marching scheme by adopting the recently developed stabilized-SAV approach $[4,19]$ where an extra crucial linear stabilization term is added to improve the energy stability. Numerical results show that this particular term is very efficient for stability while the large time steps are used.

First, we denote an auxiliary nonlocal variable $u(t)$ by

$$
u(t)=\sqrt{\int_{\Omega} F(\phi) d x+B},
$$

where $B$ be a positive constant such that $\int_{\Omega} F(\phi) d x+B$ is positive (in the following numerical example, we fix $B=1$ ).

In terms with $\phi, \psi$ and the variable $u$, the total free energy (2.14) is reformulated as the following form

$$
E(u, \phi, \psi)=\int_{\Omega}\left(\frac{\epsilon^{2}}{2}|\nabla \phi|^{2}+\frac{\alpha \epsilon^{2}}{2}|\nabla \psi|^{2}\right) d x+u^{2}-B .
$$

Then, in terms of $\phi, \psi$ and $u$, the PDE system (2.6a)-(2.6b) is further rewritten to be the following,

$$
\begin{aligned}
& \frac{1}{M} \phi_{t}-\epsilon^{2} \Delta \phi+u \bar{H}+\alpha \epsilon^{2} \psi=0, \\
& u_{t}=\frac{1}{2} \int_{\Omega} H(\phi) \phi_{t} d x, \\
& \psi=(-\Delta)^{-1}(\phi-\bar{\phi}),
\end{aligned}
$$

where the third equation above is obtained by taking the time derivative of $u(t)$, and

$$
H(\phi)=\frac{f(\phi)}{\sqrt{\int_{\Omega} F(\phi) d x+B}}, \quad \bar{H}(\phi)=H(\phi)-\frac{1}{|\Omega|} \int_{\Omega} H(\phi) d x .
$$

The initial conditions for the new system (3.3a)-(3.3c) read as follows,

$$
\phi(t=0)=\phi^{0}, \quad u(t=0)=\sqrt{\int_{\Omega} F\left(\phi^{0}\right) d x+B} .
$$

For a given positive integer $N$, set $t^{n}=n \delta t, 0 \leq n \leq N$, here $\delta t=T / N$ be the time step size. For any $\phi(x)$ and $\psi(\boldsymbol{x})$, we define the standard $L^{2}$ inner product $(\phi(\boldsymbol{x}), \psi(\boldsymbol{x}))=$ $\int_{\Omega} \phi(\boldsymbol{x}) \psi(\boldsymbol{x}) d \boldsymbol{x}$, and norm $\|\phi\|^{2}=(\phi, \phi)$. Let $\psi^{n}$ be the numerical approximation to the analytic function $\left.\psi(\cdot, t)\right|_{t=t^{n}}$.

Using the second-order backward differentiation formula (BDF2), the semi-discrete scheme for the system (3.3a)-(3.3c) is proposed as 
Given $\phi^{n}, u^{n}$ and $\phi^{n-1}, u^{n-1}$, we can update $\phi^{n+1}, u^{n+1}$ via

$$
\begin{aligned}
& \frac{3 \phi^{n+1}-4 \phi^{n}+\phi^{n-1}}{2 M \delta t}-\epsilon^{2} \Delta \phi^{n+1}+u^{n+1} \bar{H}^{*, n+1}+\alpha \epsilon^{2} \psi^{n+1}+S\left(\phi^{n+1}-\phi^{*, n+1}\right)=0, \\
& 3 u^{n+1}-4 u^{n}+u^{n-1}=\frac{1}{2} \int_{\Omega} H^{*, n+1}\left(3 \phi^{n+1}-4 \phi^{n}+\phi^{n-1}\right) d x, \\
& \psi^{n+1}=(-\Delta)^{-1}\left(\phi^{n+1}-\bar{\phi}^{n+1}\right),
\end{aligned}
$$

where

$$
\begin{aligned}
& \phi^{*, n+1}=2 \phi^{n}-\phi^{n-1}, \quad H^{*, n+1}=H\left(\phi^{*, n+1}\right), \\
& \bar{H}^{*, n+1}=H^{*, n+1}-\frac{1}{|\Omega|} \int_{\Omega} H^{*, n+1} d x,
\end{aligned}
$$

and the term associated with $S$ is an extra stabilization term.

Remark 3.1. The linear term $S\left(\phi^{n+1}-\phi^{*, n+1}\right)$ is added in the scheme as an extra stabilization term. Note this term introduces an error which is of the order $S \delta t^{2} \phi_{t t}(\cdot)$ that is comparable with the error introduced by the linear extrapolation of the nonlinear function $f(\phi)$. While adopting large time steps, it is shown by numerous examples that the stabilization term is the key term to enhance the stability for high stiffness case, cf. Fig. 1 and Fig. 2 where we give the detailed comparisons in the accuracy and stability computed by using the stabilized SAV scheme $(S \neq 0$ in the scheme (3.6a)-(3.6c)) and the non-stabilized $\operatorname{SAV}(S=0$ in the scheme (3.6a)-(3.6c)).

Remark 3.2. The computations of second-order time-discrete method (3.6a)-(3.6c) need the values of $\phi^{1}, u^{1}$. In practice, we obtain these two values by the following first-order scheme that reads as,

$$
\begin{aligned}
& \frac{\phi^{1}-\phi^{0}}{M \delta t}-\epsilon^{2} \Delta \phi^{1}+u^{1} \bar{H}^{0}+\alpha \epsilon^{2} \psi^{1}+S\left(\phi^{1}-\phi^{0}\right)=0, \\
& u^{1}-u^{0}=\frac{1}{2} \int_{\Omega} H^{0}\left(\phi^{1}-\phi^{0}\right) d x, \\
& \psi^{1}=(-\Delta)^{-1}\left(\phi^{1}-\bar{\phi}^{1}\right),
\end{aligned}
$$

where

$$
H^{0}=H\left(\phi^{0}\right), \quad \bar{H}^{0}=H^{0}-\frac{1}{|\Omega|} \int_{\Omega} H^{0} d x .
$$

By taking the $L^{2}$ inner product of (3.8a) with 1 , we derive $\bar{\phi}^{1}=\bar{\phi}^{0}$. Hence, by taking the $L^{2}$ inner product of (3.6a) with 1 , we derive

$$
\bar{\phi}^{n+1}=\bar{\phi}^{n}=\cdots=\bar{\phi}^{0},
$$

which implies that the volume fraction of the numerical solution $\phi^{n}$ is always conserved precisely. 
We now discuss the practical implementation procedure. Apparently, even with the linear property, the scheme (3.6a)-(3.6c) is still hard to solve since it is a coupled and nonlocal system for $\phi^{n+1}, \psi^{n+1}$, and $u^{n+1}$, which needs costly iterative solvers to compute at each time step. To overcome this difficulty, we introduce the following decoupling procedure that also removes the computations of nonlocal terms efficiently.

We first rewrite (3.6c) as follows:

$$
u^{n+1}=\frac{1}{2} \int_{\Omega} H^{*, n+1} \phi^{n+1} d x+g^{n},
$$

where

$$
g^{n}=\frac{4 u^{n}-u^{n-1}}{3}-\frac{1}{2} \int_{\Omega} H^{*, n+1} \frac{4 \phi^{n}-\phi^{n-1}}{3} d x .
$$

Then the numerical scheme (3.6a)-(3.6b) can be combined together to be

$$
\begin{aligned}
& \frac{3}{2 M \delta t} \phi^{n+1}+S \phi^{n+1}-\epsilon^{2} \Delta \phi^{n+1}+\alpha \epsilon^{2}(-\Delta)^{-1}\left(\phi^{n+1}-\bar{\phi}^{n+1}\right) \\
& +\frac{1}{2} \bar{H}^{*, n+1} \int_{\Omega} H^{*, n+1} \phi^{n+1} d x=\tilde{g}^{n}
\end{aligned}
$$

where $\tilde{g}^{n}$ is an explicit term defined as

$$
\tilde{g}^{n}=\frac{4 \phi^{n}-\phi^{n-1}}{2 M \delta t}+S \phi^{*, n+1}-g^{n} \bar{H}^{*, n+1} .
$$

By taking $L^{2}$ inner product of (3.12) with 1 , we obtain the explicit formula for $\bar{\phi}^{n+1}$ that reads as

$$
\bar{\phi}^{n+1}=\frac{1}{\left(\frac{3}{2 M \delta t}+S\right)|\Omega|} \int_{\Omega} \tilde{g}^{n} d x .
$$

By utilizing the operator $-\Delta$ to (3.12), we have

$$
\begin{aligned}
& -\left(\frac{3}{2 M \delta t}+S\right) \Delta \phi^{n+1}+\epsilon^{2} \Delta^{2} \phi^{n+1}+\alpha \epsilon^{2} \phi^{n+1} \\
& -\frac{1}{2} \Delta \bar{H}^{*, n+1} \int_{\Omega} H^{*, n+1} \phi^{n+1} d x=-\Delta \tilde{g}^{n}+\alpha \epsilon^{2} \bar{\phi}^{n+1} .
\end{aligned}
$$

Thus, (3.15) can be rewritten as

$$
\mathscr{P}\left(\phi^{n+1}\right)-\frac{1}{2} \Delta \bar{H}^{*, n+1} \int_{\Omega} H^{*, n+1} \phi^{n+1} d x=\widehat{g}^{n},
$$

here $\mathscr{P}(\cdot)$ is the linear operator that can be denoted as follows

$$
\mathscr{P}(\psi)=\left(-\left(\frac{3}{2 M \delta t}+S\right) \Delta+\epsilon^{2} \Delta^{2}+\alpha \epsilon^{2}\right) \psi,
$$


and $\widehat{g}^{n}=-\Delta \tilde{g}^{n}+\alpha \epsilon^{2} \bar{\phi}^{n+1}$.

Define a linear operator $\mathscr{P}^{-1}(\cdot)$, such that for any periodic function $\phi \in L^{2}(\Omega), \psi=$ $\mathscr{P}^{-1}(\phi)$ be the solution of the linear system

$$
\mathscr{P}(\psi)=\phi,
$$

with periodic boundary conditions. Applying $\mathscr{P}^{-1}$ to (3.16), we have

$$
\phi^{n+1}-\frac{1}{2} \mathscr{P}^{-1}\left(\Delta \bar{H}^{*, n+1}\right) \int_{\Omega} H^{*, n+1} \phi^{n+1} d x=\mathscr{P}^{-1}\left(\widehat{g}^{n}\right) .
$$

Taking the inner product of (3.19) with $H^{*, n+1}$, we find

$$
\int_{\Omega} H^{*, n+1} \phi^{n+1} d x=\frac{\int_{\Omega} H^{*, n+1} \mathscr{P}^{-1}\left(\widehat{g}^{n}\right) d x}{1-\frac{1}{2} \int_{\Omega} H^{*, n+1} \mathscr{P}^{-1}\left(\Delta \bar{H}^{*, n+1}\right) d x} .
$$

It is not difficult to find

$$
-\int_{\Omega} H^{*, n+1} \mathscr{P}^{-1}\left(\Delta \bar{H}^{*, n+1}\right) d x=-\int_{\Omega} H^{*, n+1} \mathscr{P}^{-1}\left(\Delta H^{*, n+1}\right) d x \geq 0,
$$

since $-\mathscr{P}^{-1}(\Delta)$ be a positive definite. Hence the explicit formula (3.20) is solvable.

Remarkably, Eq. (3.20) can be regarded as an explicit formulation for $\int_{\Omega} H^{*, n+1} \phi^{n+1} d x$. Thus, in computations, we first find

$$
\psi_{1}=\mathscr{P}^{-1}\left(\widehat{g}^{n}\right) \quad \text { and } \quad \psi_{2}=\mathscr{P}^{-1}\left(\Delta \bar{H}^{*, n+1}\right),
$$

this means that the following two decoupled, biharmonic needs to be solved

$$
\begin{aligned}
& \left(-\left(\frac{3}{2 M \delta t}+S\right) \Delta+\epsilon^{2} \Delta^{2}+\alpha \epsilon^{2}\right) \psi_{1}=\widehat{g}^{n} \\
& \left(-\left(\frac{3}{2 M \delta t}+S\right) \Delta+\epsilon^{2} \Delta^{2}+\alpha \epsilon^{2}\right) \psi_{2}=\Delta \bar{H}^{*, n+1}
\end{aligned}
$$

subject to the periodic boundary conditions. Then, using (3.20) to compute the nonlocal term $\int_{\Omega} H^{*, n+1} \phi^{n+1} d x$, we can get $\phi^{n+1}$ from (3.19) directly.

From the above implementation process, we see that the total cost at each time step is just solving two decoupled type fourth-order equations with constant coefficients,

$$
\psi_{1}=\mathscr{P}^{-1}\left(\widehat{g}^{n}\right) \quad \text { and } \quad \psi_{2}=\mathscr{P}^{-1}\left(\Delta \bar{H}^{*, n+1}\right) .
$$

Since equations with the periodic boundary conditions can be easily handled by using the Spectral method with Fourier basis. Thus, the numerical scheme is highly efficient with easy implementation process.

The unconditional energy stability of the numerical scheme (3.6a)-(3.6c) is shown as follows. 
Theorem 3.1. The time-discrete scheme (3.6a)-(3.6c) follows the discrete energy dissipation law as,

$$
\frac{1}{\delta t}\left(E^{n+1}-E^{n}\right) \leq-\frac{1}{M}\left\|\frac{3 \phi^{n+1}-4 \phi^{n}+\phi^{n-1}}{2 \delta t}\right\|^{2} \leq 0,
$$

where

$$
\begin{aligned}
E^{n+1}= & \frac{\epsilon^{2}}{2}\left(\frac{\left\|\nabla \phi^{n+1}\right\|^{2}+\left\|2 \nabla \phi^{n+1}-\nabla \phi^{n}\right\|^{2}}{2}\right)+\frac{\alpha \epsilon^{2}}{2}\left(\frac{\left\|\nabla \psi^{n+1}\right\|^{2}+\left\|2 \nabla \psi^{n+1}-\nabla \psi^{n}\right\|^{2}}{2}\right) \\
& +\frac{\left(u^{n+1}\right)^{2}+\left(2 u^{n+1}-u^{n}\right)^{2}}{2}+S \frac{\left\|\phi^{n+1}-\phi^{n}\right\|^{2}}{2}
\end{aligned}
$$

Proof. First, by taking the $L^{2}$ inner product of (3.6a) with $3 \phi^{n+1}-4 \phi^{n}+\phi^{n-1}$, we obtain

$$
\begin{aligned}
& \frac{1}{2 M \delta t}\left\|3 \phi^{n+1}-4 \phi^{n}+\phi^{n-1}\right\|^{2}+\epsilon^{2}\left(\nabla \phi^{n+1}, \nabla\left(3 \phi^{n+1}-4 \phi^{n}+\phi^{n-1}\right)\right) \\
& \quad+\left(u^{n+1} \bar{H}^{*, n+1}, 3 \phi^{n+1}-4 \phi^{n}+\phi^{n-1}\right)+\alpha \epsilon^{2}\left(\psi^{n+1}, 3 \phi^{n+1}-4 \phi^{n}+\phi^{n-1}\right) \\
& \quad+S\left(\phi^{n+1}-\phi^{*, n+1}, 3 \phi^{n+1}-4 \phi^{n}+\phi^{n-1}\right)=0 .
\end{aligned}
$$

From (3.6c), we can derive

$$
-\Delta\left(3 \psi^{n+1}-4 \psi^{n}+\psi^{n-1}\right)=3 \phi^{n+1}-4 \phi^{n}+\phi^{n-1}-\left(3 \bar{\phi}^{n+1}-4 \bar{\phi}^{n}+\bar{\phi}^{n-1}\right) .
$$

Second, we take the $L^{2}$ inner product of (3.26) with $\alpha \epsilon^{2} \psi^{n+1}$ to get

$$
\alpha \epsilon^{2}\left(3 \nabla \psi^{n+1}-4 \nabla \psi^{n}+\nabla \psi^{n-1}, \nabla \psi^{n+1}\right)=\alpha \epsilon^{2}\left(3 \phi^{n+1}-4 \phi^{n}+\phi^{n-1}, \psi^{n+1}\right),
$$

where the term

$$
\left(3 \bar{\phi}^{n+1}-4 \bar{\phi}^{n}+\bar{\phi}^{n-1}, \psi^{n+1}\right)=0, \quad \text { since } \int_{\Omega} \psi^{n+1} d x=0 .
$$

Third, by multiplying (3.6b) with $2 u^{n+1}$, we obtain the following equality,

$$
\begin{aligned}
2\left(3 u^{n+1}-4 u^{n}+u^{n-1}\right) u^{n+1} & =u^{n+1} \int_{\Omega} H^{*, n+1}\left(3 \phi^{n+1}-4 \phi^{n}+\phi^{n-1}\right) d x \\
& =u^{n+1} \int_{\Omega} \bar{H}^{*, n+1}\left(3 \phi^{n+1}-4 \phi^{n}+\phi^{n-1}\right) d x
\end{aligned}
$$

where the validity of the latter equality is due to (3.9).

Finally, we combine (3.25), (3.27), (3.28), and apply the following two identities

$$
\begin{aligned}
& 2 a(3 a-4 b+c)=a^{2}+(2 a-b)^{2}-b^{2}-(2 b-c)^{2}+(a-2 b+c)^{2}, \\
& (3 a-4 b+c)(a-2 b+c)=(a-b)^{2}-(b-c)^{2}+2(a-2 b+c)^{2},
\end{aligned}
$$


to obtain

$$
\begin{aligned}
& \quad \frac{\epsilon^{2}}{2}\left(\left\|\nabla \phi^{n+1}\right\|^{2}+\left\|2 \nabla \phi^{n+1}-\nabla \phi^{n}\right\|^{2}\right)-\frac{\epsilon^{2}}{2}\left(\left\|\nabla \phi^{n}\right\|^{2}+\left\|2 \nabla \phi^{n}-\nabla \phi^{n-1}\right\|^{2}\right) \\
& \quad+\frac{\alpha \epsilon^{2}}{2}\left(\left\|\nabla \psi^{n+1}\right\|^{2}+\left\|2 \nabla \psi^{n+1}-\nabla \psi^{n}\right\|^{2}\right)-\frac{\alpha \epsilon^{2}}{2}\left(\left\|\nabla \psi^{n}\right\|^{2}+\left\|2 \nabla \psi^{n}-\nabla \psi^{n-1}\right\|^{2}\right) \\
& \quad+\left(\left(u^{n+1}\right)^{2}+\left(2 u^{n+1}-u^{n}\right)^{2}\right)-\left(\left(u^{n}\right)^{2}+\left(2 u^{n}-u^{n-1}\right)^{2}\right)+S\left\|\phi^{n+1}-\phi^{n}\right\|^{2} \\
& \quad-S\left\|\phi^{n}-\phi^{n-1}\right\|^{2}+\frac{\epsilon^{2}}{2}\left\|\nabla \phi^{n+1}-2 \nabla \phi^{n}+\nabla \phi^{n-1}\right\|^{2}+\frac{\alpha \epsilon^{2}}{2}\left\|\nabla \psi^{n+1}-2 \nabla \psi^{n}+\nabla \psi^{n-1}\right\|^{2} \\
& \quad+\left(u^{n+1}-2 u^{n}+u^{n-1}\right)^{2}+2 S\left\|\phi^{n+1}-2 \phi^{n}+\phi^{n-1}\right\|^{2} \\
& =-\frac{1}{2 M \delta t}\left\|3 \phi^{n+1}-4 \phi^{n}+\phi^{n-1}\right\|^{2} .
\end{aligned}
$$

Hence, the desired result is obtained after dropping some positive terms.

Remark 3.3. Note $\frac{1}{\delta t}\left(E^{n+1}-E^{n}\right)$ is actually a temporal second-order approximation of the term $\frac{d}{d t} E(u, \phi)$ at $t=t^{n+1}$. Since for any smooth variable $\psi$ with time, we always have the following heuristic approximations as

$$
\begin{aligned}
& \frac{\left\|\psi^{n+1}\right\|^{2}-\left\|2 \psi^{n+1}-\psi^{n}\right\|^{2}}{2 \delta t}-\frac{\left\|\psi^{n}\right\|^{2}-\left\|2 \psi^{n}-\psi^{n-1}\right\|^{2}}{2 \delta t} \\
\cong & \frac{\left\|\psi^{n+2}\right\|^{2}-\left\|\psi^{n}\right\|^{2}}{2 \delta t}+\mathcal{O}\left(\delta t^{2}\right) \cong \frac{d}{d t}\left\|\psi\left(t^{n+1}\right)\right\|^{2}+\mathcal{O}\left(\delta t^{2}\right),
\end{aligned}
$$

and

$$
\frac{\left\|\psi^{n+1}-\psi^{n}\right\|^{2}-\left\|\psi^{n}-\psi^{n-1}\right\|^{2}}{2 \delta t} \cong \mathcal{O}\left(\delta t^{2}\right)
$$

Remark 3.4. In this paper, we only consider the time discrete schemes. Note all the proofs are based on a variational formula and the test functions are in the same space as the space of the trial functions, thus all analytical results can be applied to other Galerkin approximations method in the space without any essential difficulties. We leave the fully discrete scheme to the interested readers.

\section{Numerical simulation}

In this section, we perform the numerical simulations in two and three-dimensional spaces to demonstrate the accuracy and energy stability of the developed stabilized-SAV scheme (3.6a)-(3.6c). We use the square 2D or 3D computed domain as $\Omega=[0, L]^{d}$ with $d=2,3$ and the space with periodic boundary conditions is then discretized by adopting the Fourier-spectral method. 


\subsection{Accuracy and stability test}

We perform simulations to test the convergence order of the stabilized-SAV scheme (3.6a)-(3.6c), defined by SSAV for short. Meanwhile, in order to show its stability and accuracy, the convergence rates of the non-stabilized version of the SAV scheme is also computed, i.e., scheme (3.6a)-(3.6c) where $S=0$, denoted by SAV for short.

We perform refinement tests for temporal convergence. The initial conditions (two adjacent circles) are set as follows,

$$
\phi(x, y, t=0)=\frac{1}{4} \sum_{i=1}^{2}-\tanh \left(\frac{\sqrt{\left(x-x_{i}\right)^{2}+\left(y-y_{i}\right)^{2}-r_{i}}}{1.5 \epsilon}\right)+\frac{3}{4},
$$

where $\left(x_{1}, y_{1}, r_{1}\right)=(\pi-0.8, \pi, 1.4)$ and $\left(x_{2}, y_{2}, r_{2}\right)=(\pi+1.7, \pi, 0.5)$. We set the model parameters to be $\epsilon=0.06, S=2, B=1$. The accuracy tests are shown by varying two parameters: the mobility parameter $M$ and nonlocal parameter $\alpha$.

We discretize the $2 \mathrm{D}$ square domain $\Omega=[0,2 \pi]^{2}$ by using $N_{x}=N_{y}=128$ Fourier-modes for spatial direction. Therefore, the errors from the spatial direction can be ignored, while the time discretization errors are dominant.

The errors are computed by using the solution obtained with a very tiny time step size $\delta t=1 \mathrm{e}-9$ computed by the scheme SSAV as the exact solution for computing errors since the exact solution is not known. By varying the time step sizes, we show the $L^{2}$ errors of the phase function $\phi$ between the exact and approximate solutions at time $t=10$.

First, we will test the convergence rates for the low stiffness case, where we use small mobility and nonlocal parameters as $M=1, \alpha=0.01$. In Fig. 1(a), we plotted the $L^{2}$ errors that are calculated by the two schemes SSAV and SAV. From the accuracy plots, we

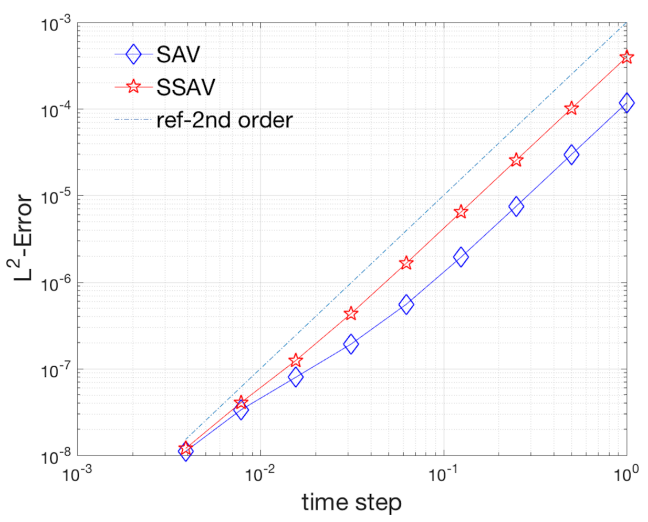

(a) $M=1, \alpha=0.01$

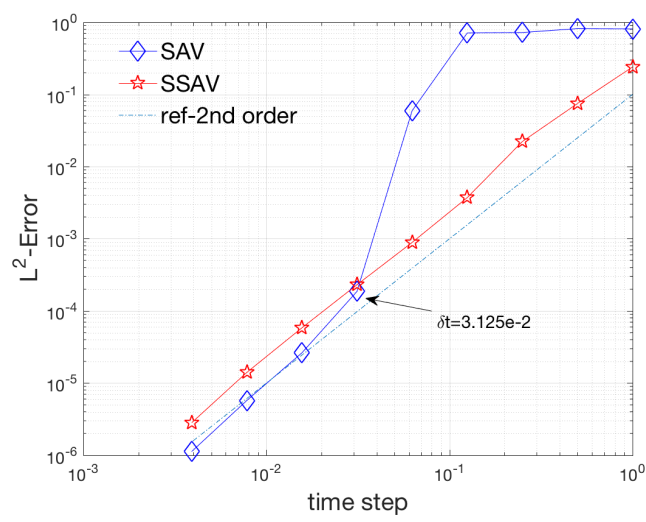

(b) $M=100, \alpha=100$

Figure 1: The $L^{2}$ numerical errors for the phase-field variable $\phi$ that are computed by using the schemes SSAV and SAV using various temporal resolutions, where the parameters are set as (a) $M=1, \alpha=0.01$ (low stiffness case); (b) $M=100, \alpha=100$ (high stiffness case). 


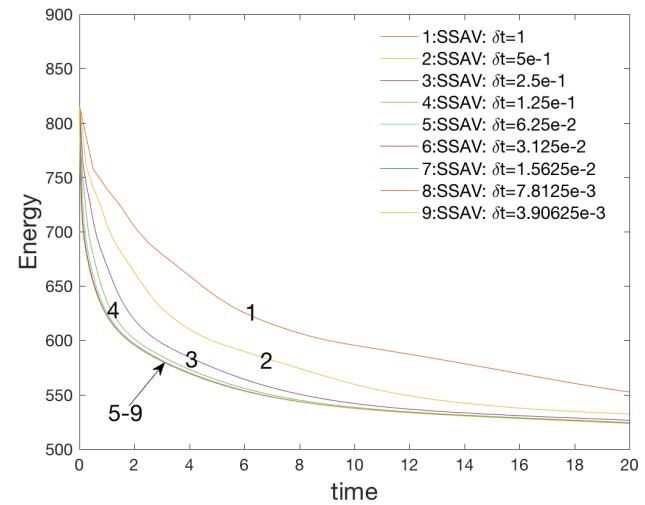

(a) Energy evolution by using the stabilized scheme SSAV

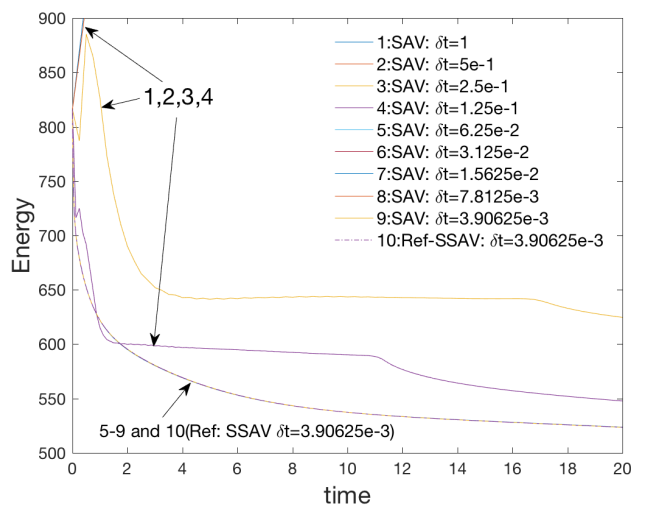

(b) Energy evolution by using the non-stabilized scheme SAV

Figure 2: High stiffness case: time evolution of the free energy functional (2.14) using various time step sizes computed by the two schemes SSAV and its non-stabilized version SAV, where the parameters are $M=100$, $\alpha=100$.

find that the performances of these two schemes are both very good and the accuracies almost perfectly match the second-order accuracy line. This implies that the stabilizer term is actually not necessary for the low stiffness case. Second, we test the convergence rates for the high stiffness case, where we set $M=100, \alpha=100$. The errors in $L^{2}$ norm are plotted in Fig. 1(b). When $\delta t>3.125 \mathrm{e}-2$, we note the accuracy is totally destroyed since the non-stabilized scheme is not stable. When $\delta t \leq 3.125 \mathrm{e}-2$, the non-stabilized scheme SAV starts to present the second-order accuracy. On the contrary, we observe that the stabilized scheme SSAV always show the second-order accuracy since the stability is well controlled.

Finally, we investigate whether the stabilized scheme SSAV is unconditionally energy stable by computing various energy evolution curves with different time steps. We still use the high stiffness case of large mobility and nonlocal parameters as $M=100, \alpha=100$. In Fig. 2, the evolution profile of the free energy(2.14) is calculated by schemes SSAV and SAV. For all test steps, the energy curves calculated by SSAV scheme show monotonic attenuation, which indicates that our numerical scheme is unconditionally stable, shown in Fig. 2(a). In contrast, the total energy calculated by the standard SAV method only decays when $\delta t$ is very small $(\delta t \leq 6.25 \mathrm{e}-2)$. When the time step $\delta t>6.25 \mathrm{e}-2$, the energy does not decay, which implies the non-stabilized scheme actually performs bad stability when large time steps are used.

We summarize all accuracy and stability tests to conclude that, (i) if the stiffness is low, the stabilizer term is not necessary and both of the stabilized or non-stabilized schemes can solve the model well; (ii) if the stiffness is high, the stability and accuracy tests show the stabilized scheme is far more superior to the non-stabilized scheme while using large time steps. 

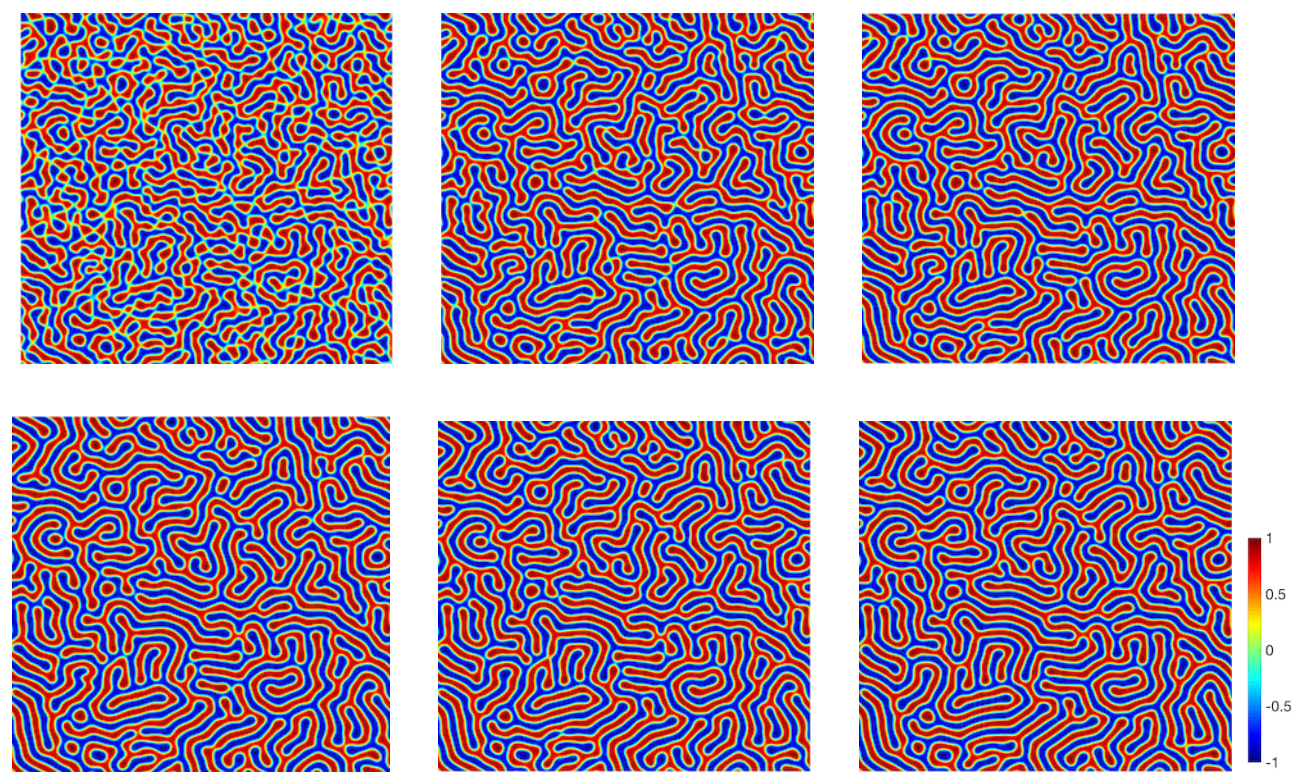

Figure 3: The pattern evolution of the phase variable $\phi$ in $2 \mathrm{D}$ where $\tilde{\phi}=0$ and $\delta t=0.01$. Snapshots of the numerical approximation are taken at $t=20,40,60,80,100$, and 200.
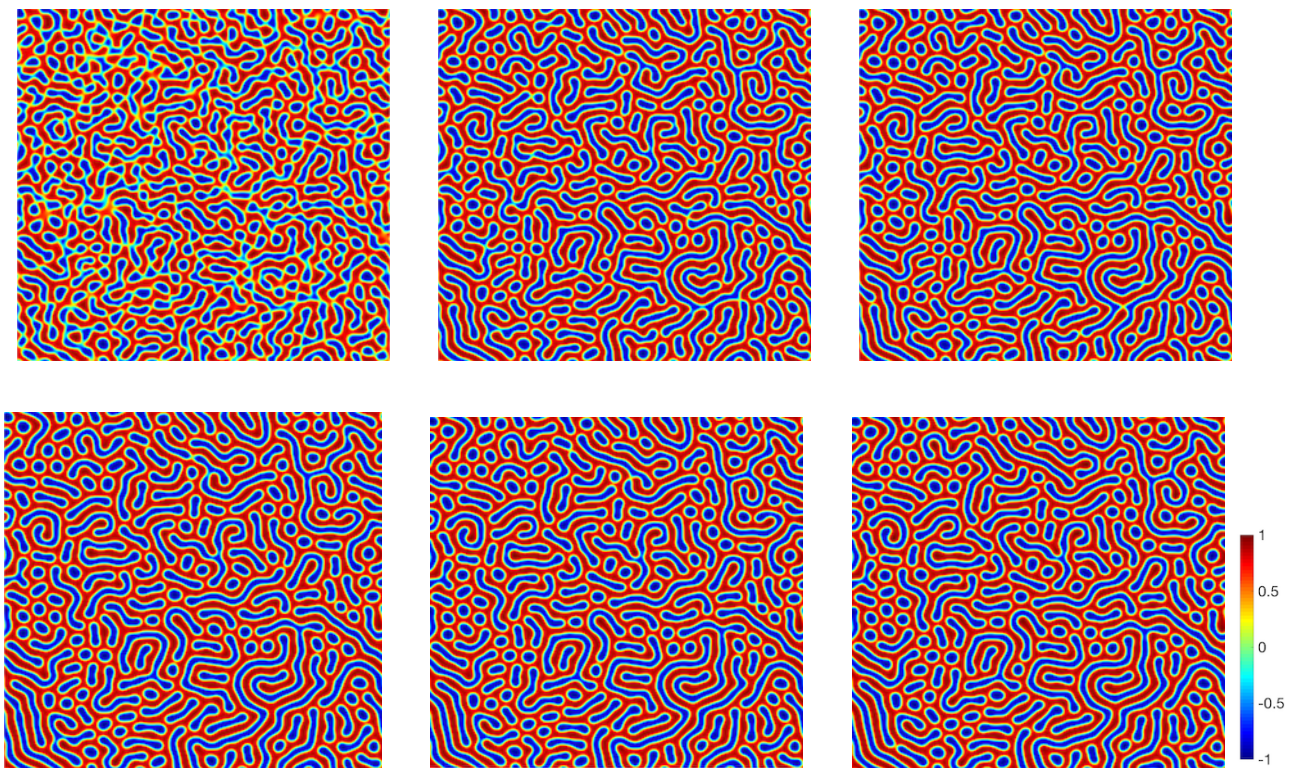

Figure 4: The pattern evolution of the phase variable $\phi$ in $2 \mathrm{D}$ where $\tilde{\phi}=0.1$ and $\delta t=0.01$. Snapshots of the numerical approximation are taken at $t=20,40,60,80,100$, and 200.

\subsection{Spinodal Decomposition}

In the following example, we will study the spinodal decomposition via the developed 

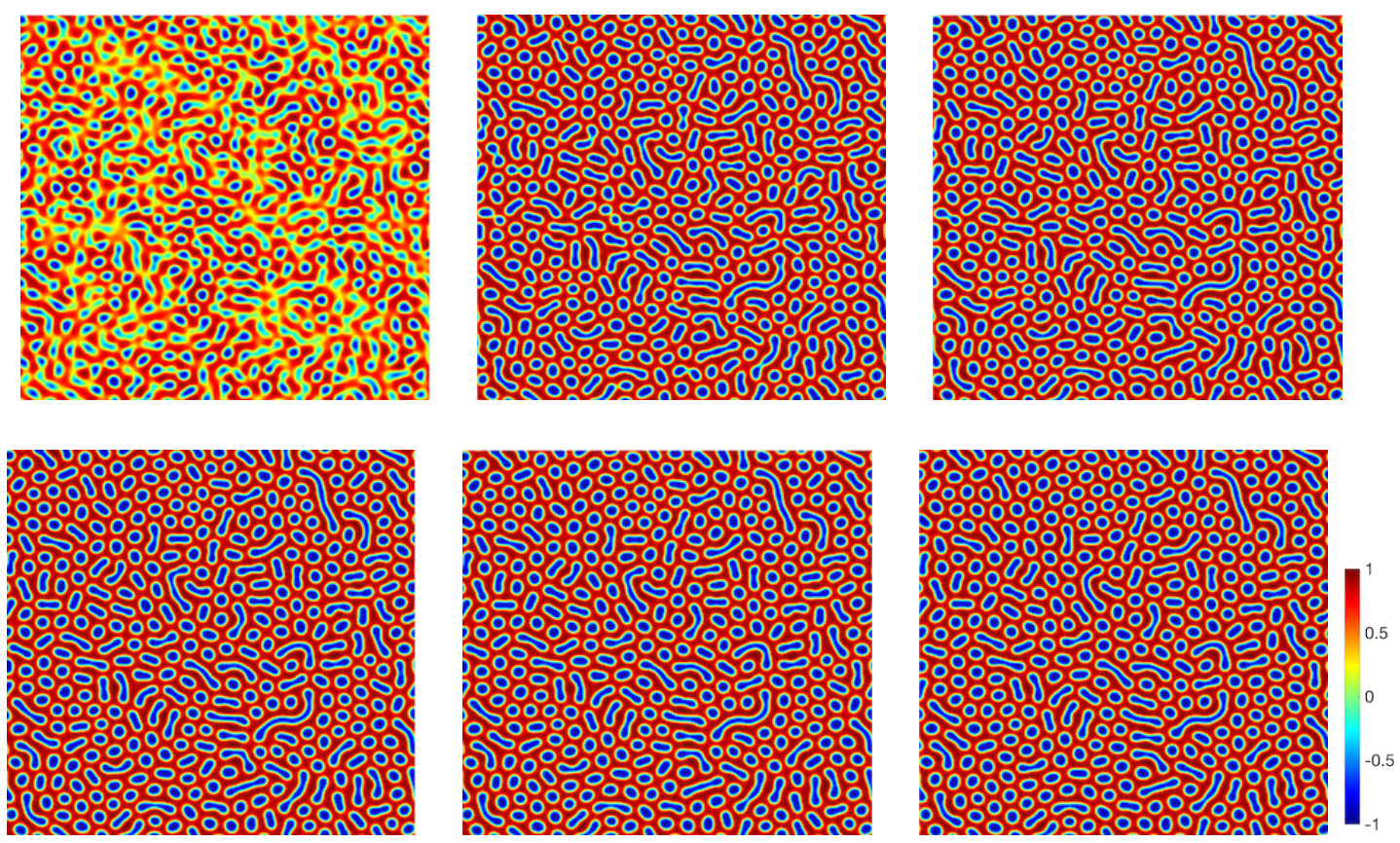

Figure 5: The pattern evolution of the phase variable $\phi$ in 2D where $\tilde{\phi}=0.2$ and $\delta t=0.01$. Snapshots of the numerical approximation are taken at $t=20,40,60,80,100$, and 200.

scheme SSAV. By setting a perturbed homogeneous binary mixture as the initial condition, the homogeneous structure finally evolves to the two-phase state due to the spontaneous growth of the concentration fluctuations.

\subsubsection{D case}

We set the initial condition as the randomly perturbed concentration field as follows,

$$
\phi(x, y, t=0)=\tilde{\phi}+0.001 \mathrm{rand}(x, y),
$$

where the rand $(x, y)$ is the random number in $[-1,1]$ that follows the normal distribution. We use the stabilized scheme SSAV with the time step $\delta t=0.01$ and discretize the domain $[0,2 \pi]^{2}$ using $N_{x}=N_{y}=256$ Fourier modes. The other model parameters are set as $\alpha=$ $300000, \epsilon=0.02, M=1, S=2, B=1$.

In Fig. 3, we perform numerical simulations for the initial value $\tilde{\phi}=0$ and snapshots of the phase-field variable $\phi(x, t)$ are taken at $t=20,40,60,80,100$, and 200. We observe the final equilibrium solution forms the cylindrical phase where the blue region is entangled with the red region everywhere. In Fig. 4 , by using $\tilde{\phi}=0.1$, we observe that a small quantity of the blue region starts to form the BCC phase and most of the blue region still graft and entangle with the red region. When the initial value is $\tilde{\phi}=0.2$, illustrated in Fig. 5, we observe that more blue regions form the BCC phase and a small quantity of it 

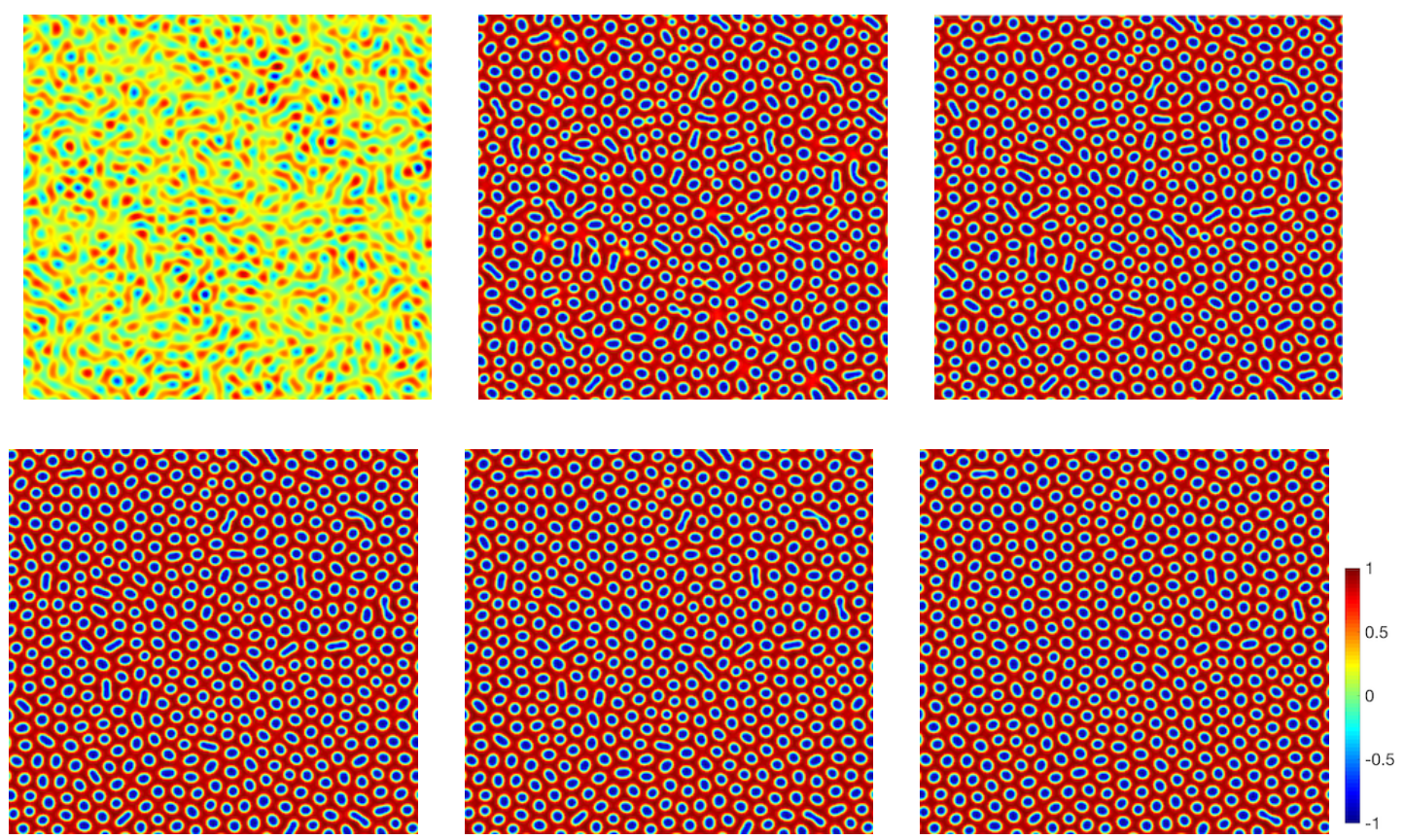

Figure 6: The pattern evolution of the phase variable $\phi$ in $2 \mathrm{D}$ where $\tilde{\phi}=0.3$ and $\delta t=0.01$. Snapshots of the numerical approximation are taken at $t=20,40,60,80,100$, and 200.

still forms the cylindrical phase. Finally, by using $\tilde{\phi}=0.3$, presented in Fig. 6, we observe that the final equilibrium solution turns to be the pure BCC phase in the whole domain.

In Fig. 7, through the side by side comparisons between the final equilibrium numerical solutions of the above four cases, the equilibrium solution by using the Cahn-Hilliard model (2.15a)-(2.15c) with the same initial values and parameters, and the experiments for block copolymers in the thin films (cf. [30]), we can see that the numerical simulations of the Allen-Cahn model are consistent with Cahn-Hilliard model in quality, and both of them are consistent with the experimental results.

In Fig. 8, we present the evolution of the total free energy functional (2.14) for all four cases with initial values of $\tilde{\phi}=0,0.1,0.2$, and 0.3 . The energy curves show the decays with the time that confirms that the scheme SSAV is unconditionally stable.

\subsubsection{D case}

We set the initial profile of concentration field as follows

$$
\phi(x, y, z, t=0)=\tilde{\phi}+0.001 \operatorname{rand}(x, y, z),
$$

where the rand $(x, y, z)$ is the random number in $[-1,1]$ that follows the normal distribution. We use the scheme SSAV with the time step $\delta t=0.01$ and discretize the space using 

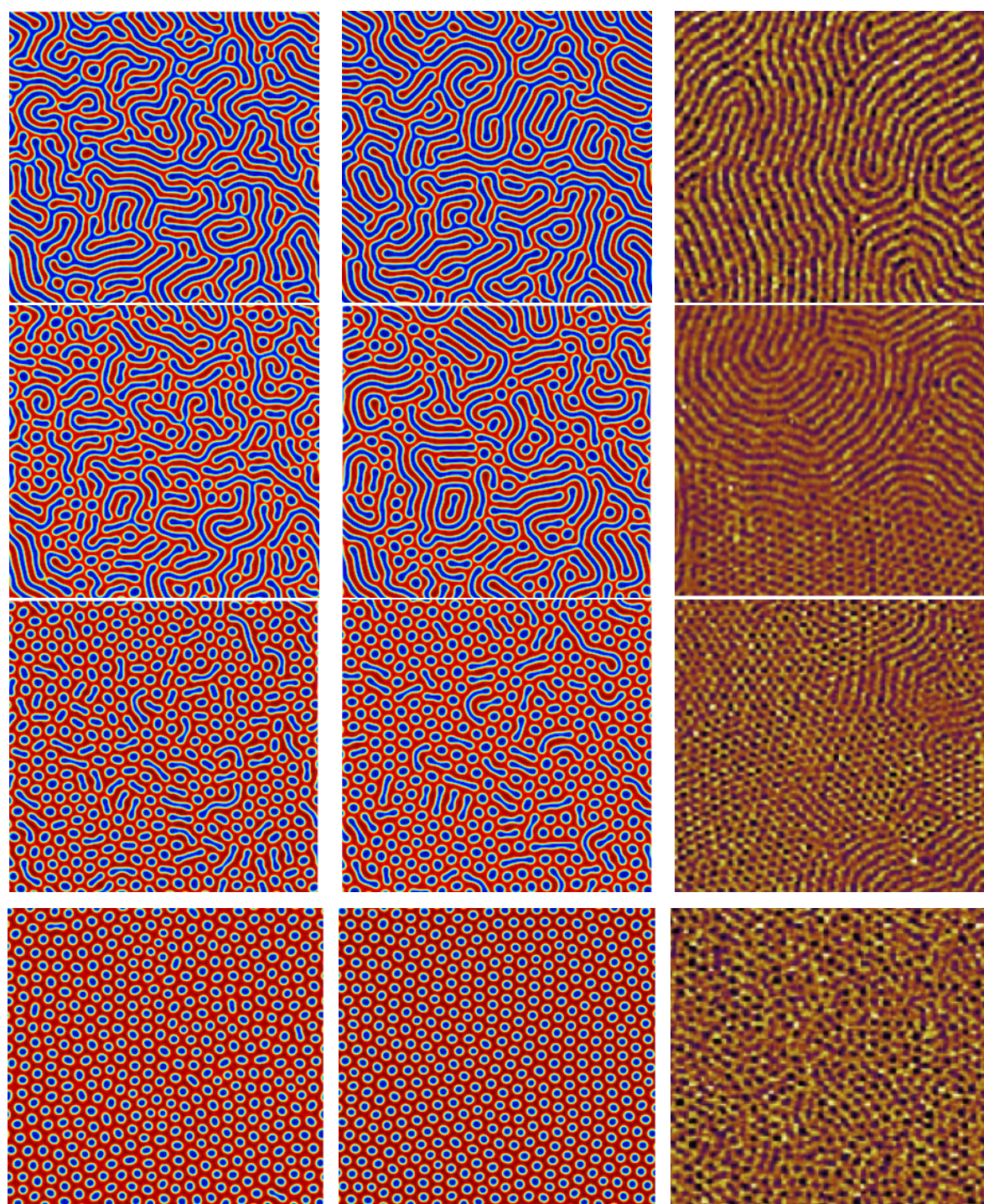

(a) Allen-Cahn model

(b) Cahn-Hilliard model

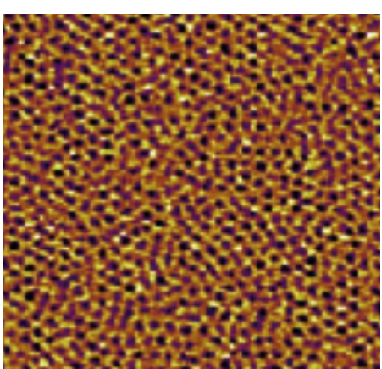

(c) Experiment

Figure 7: Qualitative comparisons of the equilibrium solutions computed by using the new Allen-Cahn model (2.7a)-(2.7b), the classical Cahn-Hilliard model (2.15a)-(2.15c), and the experimental benchmark of block copolymers in the thin film (cf. [30]). The left panel (a) is the new Allen-Cahn model, the middle panel (b) is the Cahn-Hilliard model, and the right panel (c) is the experimental results from [30]. From the top panel to the bottom one, the initial values are $\tilde{\phi}=0,0.1,0.2$, and 0.3 .

$N_{x}=N_{y}=N_{z}=128$ Fourier modes. The computed domain is $[0,2]^{3}$ and the parameters are $\alpha=300000, \epsilon=0.02, M=1, S=2, B=1$.

In Figs. 9, 10, and 11, we set the initial value $\tilde{\phi}=0,0.2$ and 0.4 , respectively. We plot 


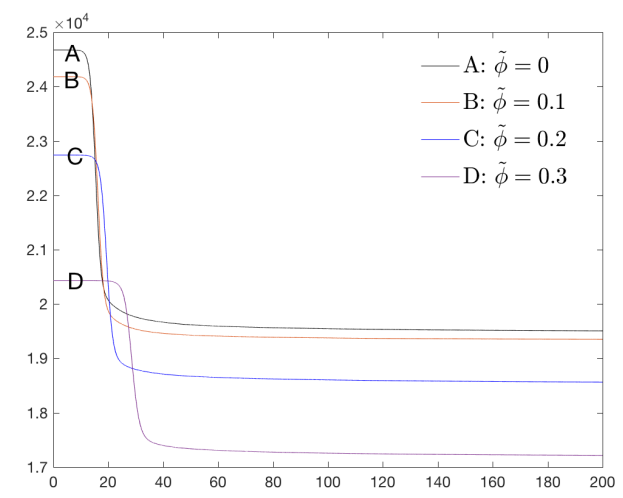

(a)

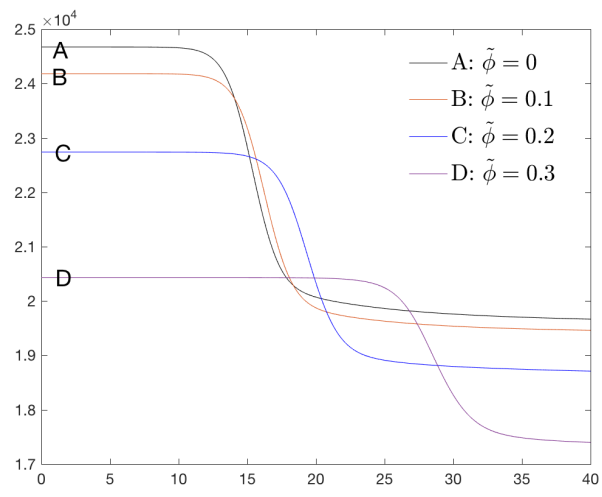

(b)

Figure 8: (a) Time evolution of the free energy functional (2.14) for the 2D spinodal decomposition example with the initial values of $\bar{\phi}=0,0.1,0.2$, and 0.3 ; (b) is the close-up view where the energies decay fast.
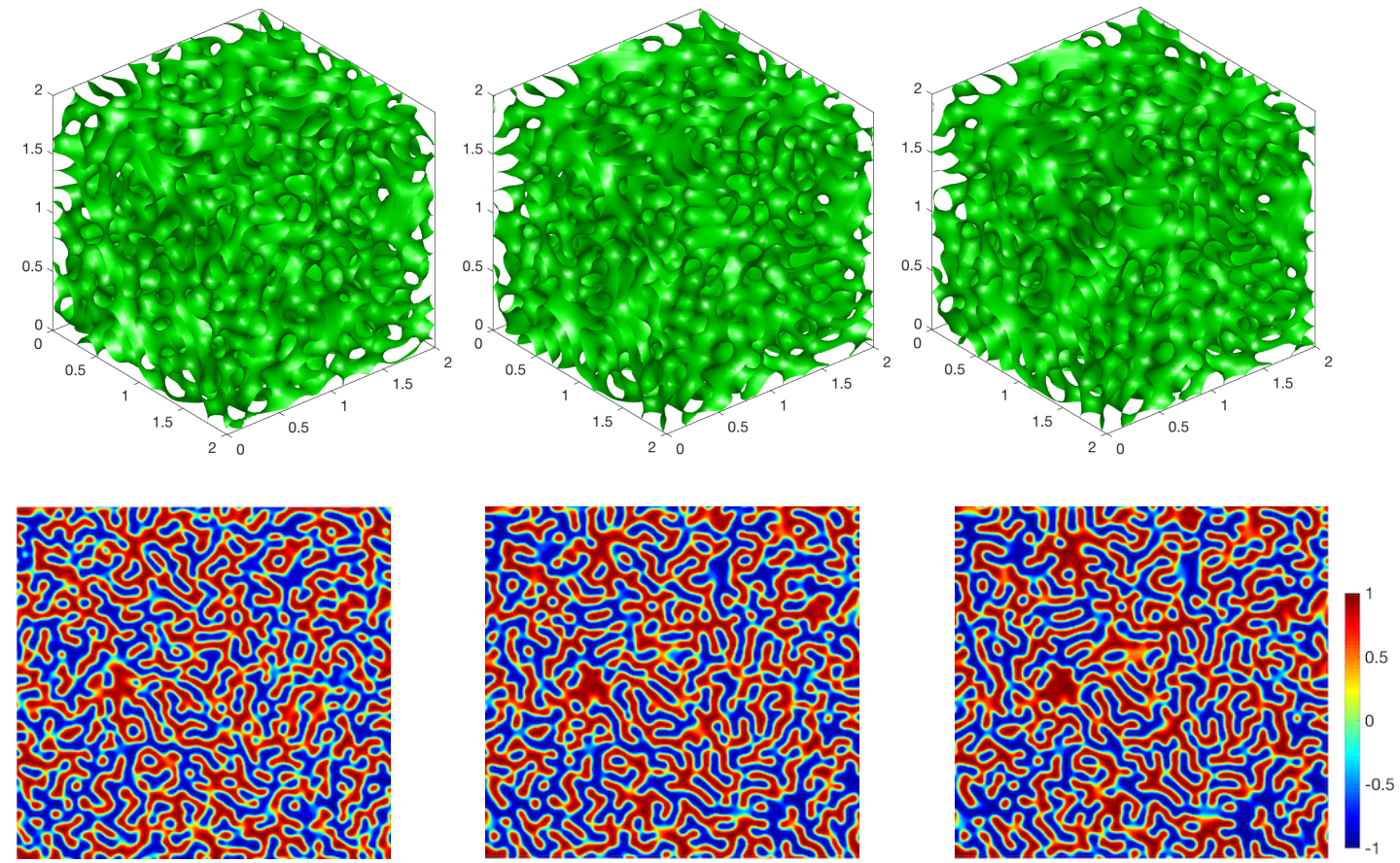

Figure 9: (a) The 3D pattern evolution of the iso-surface of the phase variable $\phi$ at $t=40,200$, and 500 with the initial condition is $\tilde{\phi}=0$ and time step $\delta t=0.01$; (b) $2 \mathrm{D}$ cross-section of $\phi(1, \cdot, \cdot)$.

the snapshots of the isosurfaces of $\{\phi(x, y, z)=0\}$ at various times. The final equilibrium solution forms the gyroidal (cylindrical) shape for $\tilde{\phi}=0$, the mixed gyroidal and spherical shapes for $\tilde{\phi}=0.2$, and the pure spherical shape for $\tilde{\phi}=0.4$. In order to gain a more accurate 

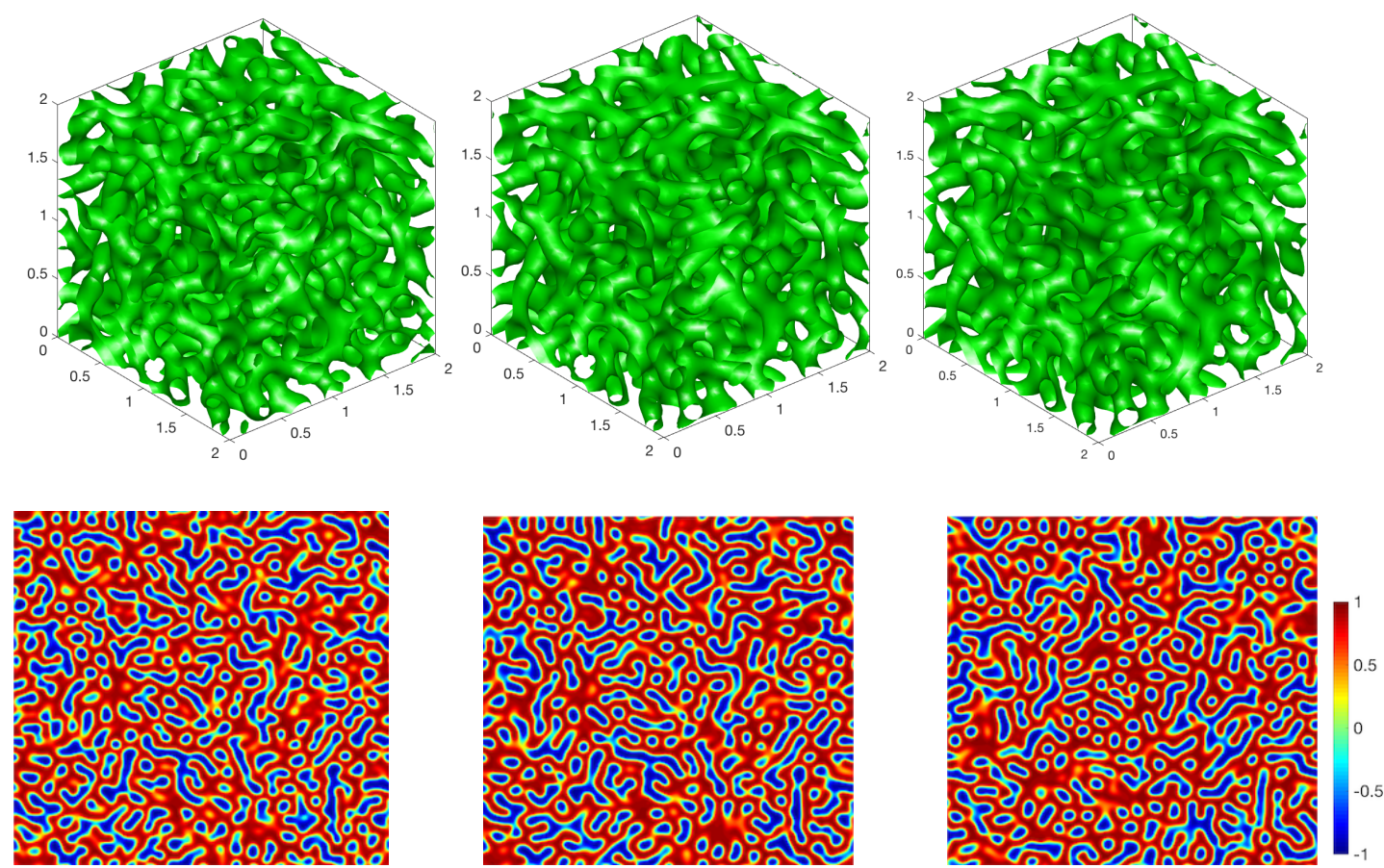

Figure 10: (a) The 3D pattern evolution of the iso-surface of the phase variable $\phi$ at $t=40,200$, and 500 with the initial condition is $\tilde{\phi}=0.2$ and time step $\delta t=0.01$; (b) 2D cross-section of $\phi(1, \cdot, \cdot)$.

perspective, the views of cut-off planes $\phi(1, \cdot, \cdot)$ of each 3D snapshot are presented. In Fig. 12, we present the evolution of the total free energy functional (2.14) for these three cases.

\subsection{D dynamics with the imposed electric field}

In this simulation, we apply an external electric field to investigate the pattern formations. The application of electric fields is an efficient approach to produce various patterns of nano-structured materials. An additional forcing term contributed by the electric field along $x$-direction is added to the model system (2.7a)-(2.7b) which reads as follows,

$$
\begin{aligned}
& \frac{1}{M} \phi_{t}-\epsilon^{2} \Delta \phi+f(\phi)+\alpha \epsilon^{2} \psi-\frac{1}{|\Omega|} \int_{\Omega} f(\phi) d x+\beta \epsilon^{2}(-\Delta)^{-1} \phi_{x x}=0, \\
& \psi=(-\Delta)^{-1}(\phi-\bar{\phi}) .
\end{aligned}
$$

Notice that the imposed electric term is linear, therefore we can simply modify (3.6a) in the SSAV scheme as

$$
\frac{3 \phi^{n+1}-4 \phi^{n}+\phi^{n-1}}{2 M \delta t}-\epsilon^{2} \Delta \phi^{n+1}+u^{n+1} \bar{H}^{*, n+1}
$$




$$
+\alpha \epsilon^{2} \psi^{n+1}+\beta \epsilon^{2}(-\Delta)^{-1} \phi_{x x}^{n+1}+S\left(\phi^{n+1}-\phi^{*, n+1}\right)=0 .
$$

The computed domain is still $[0,2 \pi]^{2}$ and the initial condition is still (4.3). We keep the model parameters $M=1, \epsilon=0.02, \alpha=300000, S=2, B=1$ and vary the initial value $\tilde{\phi}$ and the magnitude of the electric field parameter $\beta$.
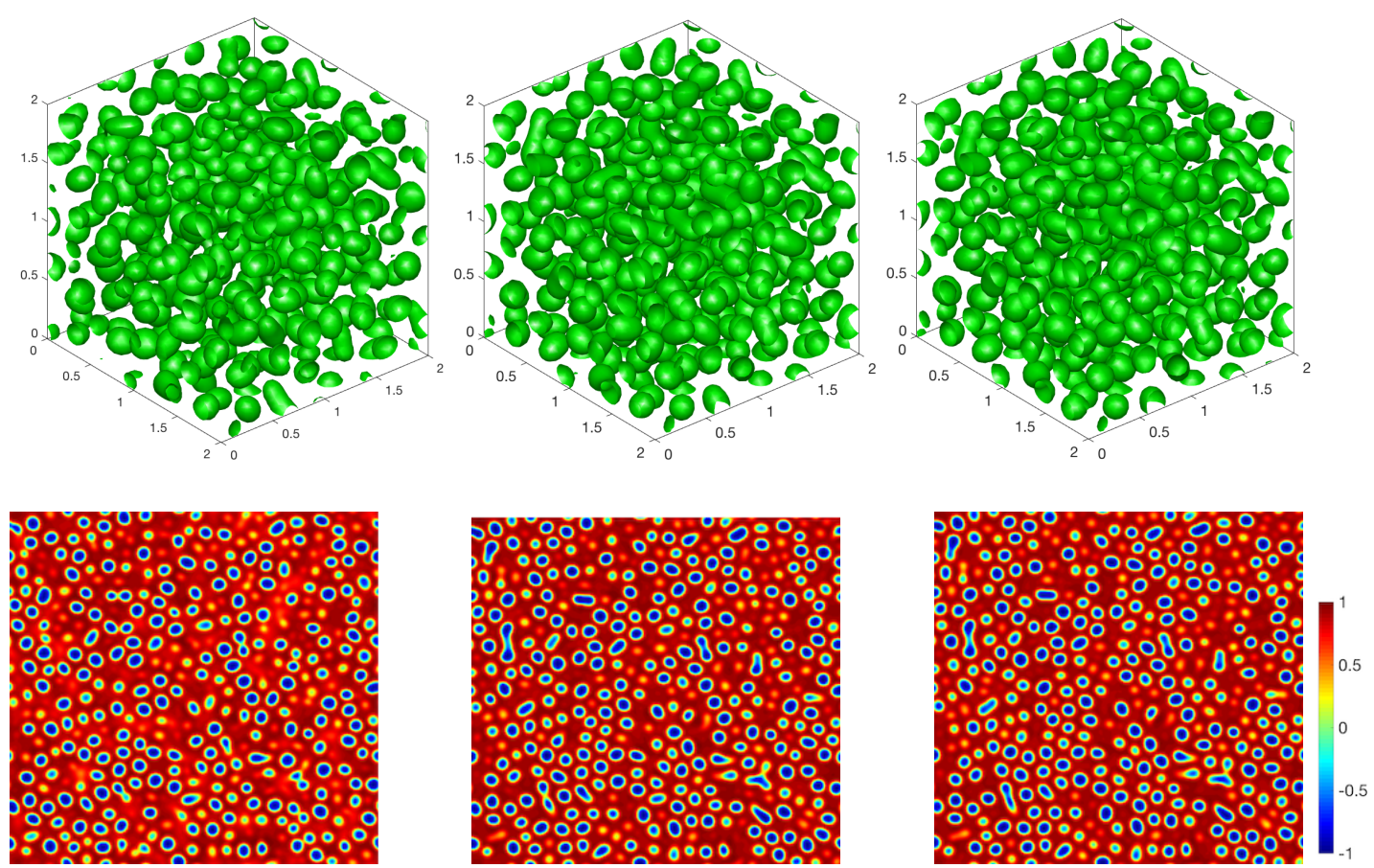

Figure 11: (a) The 3D pattern evolution of the iso-surface of the phase variable $\phi$ at $t=40,200$, and 500 with the initial condition is $\tilde{\phi}=0.4$ and time step $\delta t=0.01$; (b) $2 \mathrm{D}$ cross-section of $\phi(1, \cdot, \cdot)$.

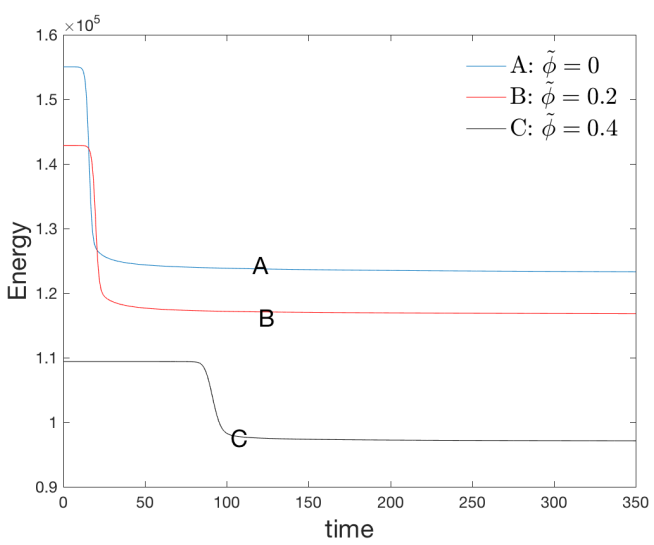

Figure 12: Time evolution of the free energy functional (2.14) for the 3D spinodal decomposition examples with the initial values of $\tilde{\phi}=0,0.2$, and 0.4 . 

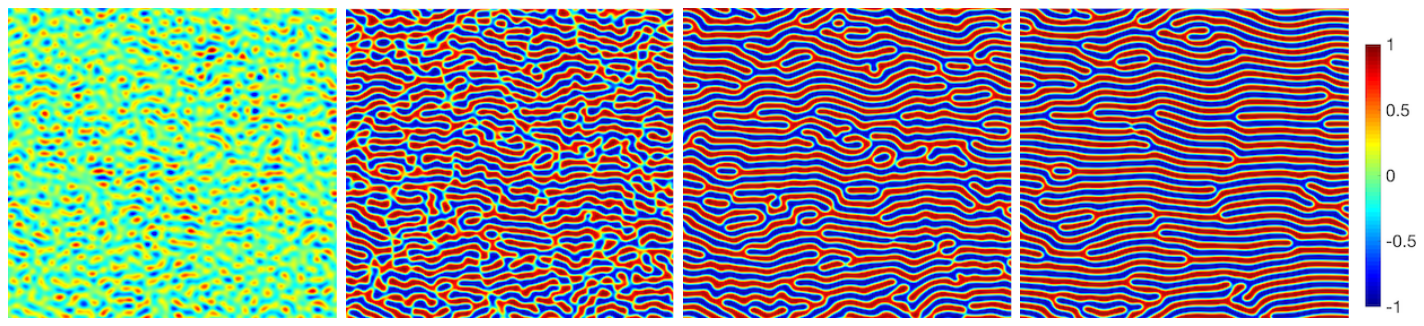

Figure 13: The pattern evolution of the phase variable $\phi$ in $2 \mathrm{D}$ where $\tilde{\phi}=0, \delta t=0.01$, and $\beta=200$. Snapshots of the numerical approximation are taken at $t=10,20,50$, and 200 .
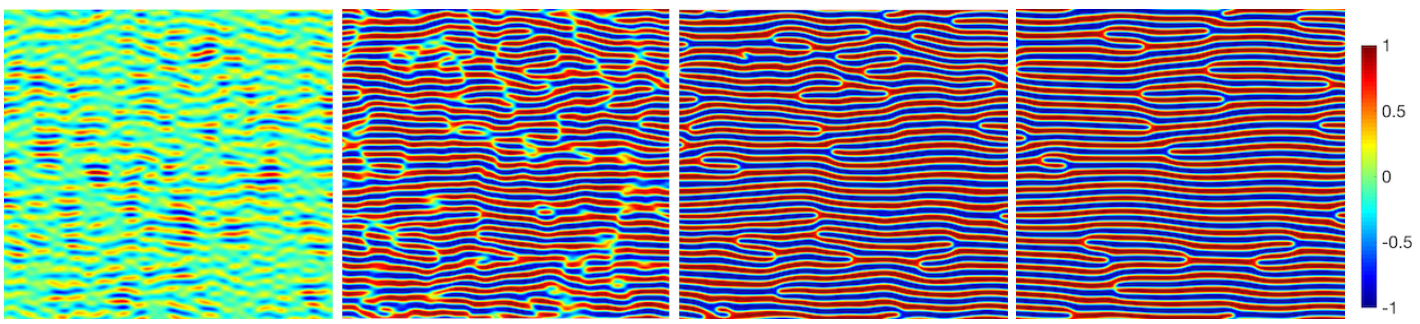

Figure 14: The pattern evolution of the phase variable $\phi$ in $2 \mathrm{D}$ where $\tilde{\phi}=0, \delta t=0.01$, and $\beta=1000$. Snapshots of the numerical approximation are taken at $t=10,20,50$, and 200.
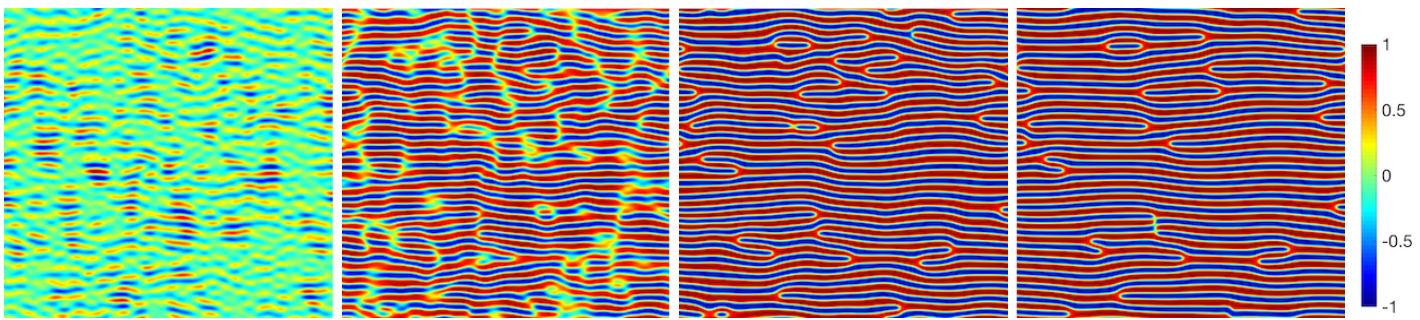

Figure 15: The pattern evolution of the phase variable $\phi$ in $2 \mathrm{D}$ where $\tilde{\phi}=0.1, \delta t=0.01$, and $\beta=1000$. Snapshots of the numerical approximation are taken at $t=10,20,50$, and 200.

First, we set $\tilde{\phi}=0$ and $\beta=200$. In Fig. 13, we observe that phase dislocations gradually disappear and curvy lamellar profiles are finally presented where a few dislocations are shown. Then by keeping the initial value of $\tilde{\phi}=0$ and increasing the magnitude of the electric field to $\beta=1000$ in Fig. 14, we observe the lamellar phase become more straight with relatively fewer dislocations. In Figs. 15, 16, and 17, we keep the strong electric field $\beta=1000$, and change the initial value from $\tilde{\phi}=0.1$ to 0.4 with the incremental value of 0.1 , we observe that various patterns of lamellar phases are obtained accordingly. All these obtained simulations are qualitatively consistent with the numerical results in $[14,22,23]$. 

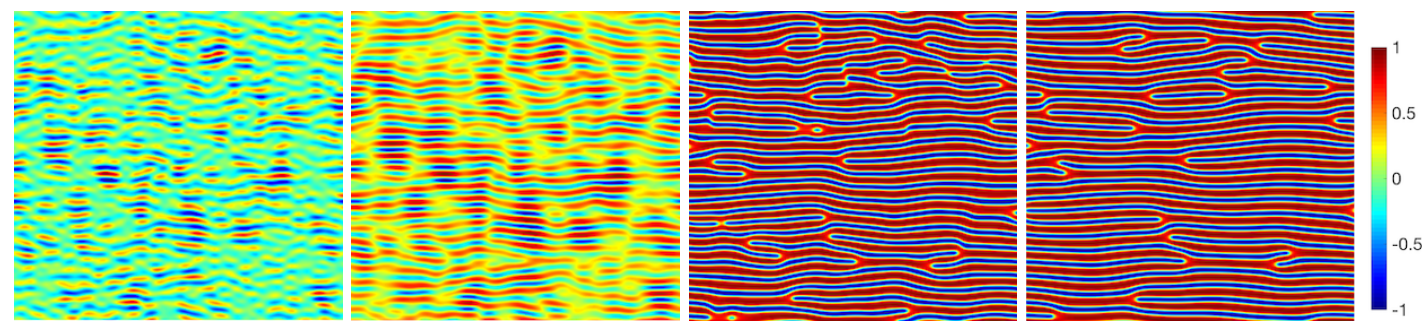

Figure 16: The pattern evolution of the phase variable $\phi$ in $2 \mathrm{D}$ where $\tilde{\phi}=0.2, \delta t=0.01$, and $\beta=1000$. Snapshots of the numerical approximation are taken at $t=10,20,50$, and 200.
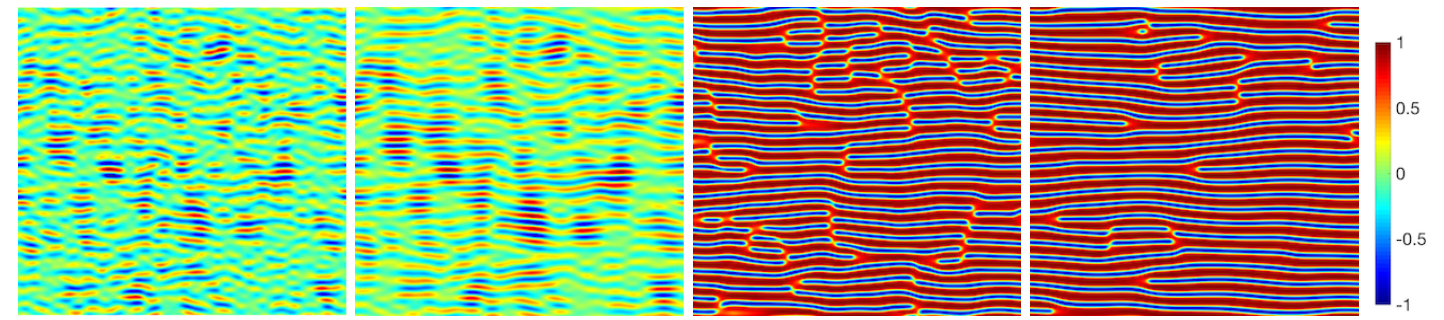

Figure 17: The pattern evolution of the phase variable $\phi$ in $2 \mathrm{D}$ where $\tilde{\phi}=0.3, \delta t=0.01$, and $\beta=1000$. Snapshots of the numerical approximation are taken at $t=10,20,50$, and 200.
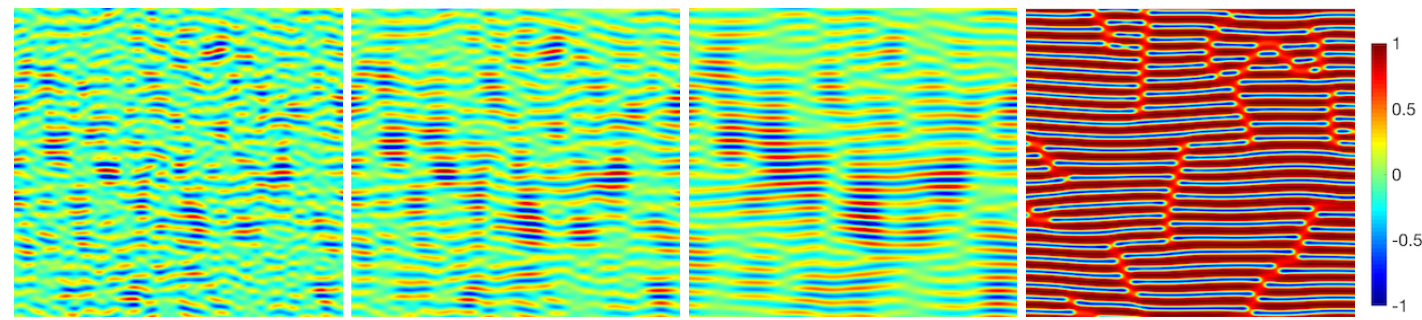

Figure 18: The pattern evolution of the phase variable $\phi$ in $2 \mathrm{D}$ where $\tilde{\phi}=0.4, \delta t=0.01$, and $\beta=1000$. Snapshots of the numerical approximation are taken at $t=10,20,50$, and 200.

\section{Concluding remarks}

In this paper, we develop a new conserved Allen-Cahn type phase-field model for diblock copolymers by using the $L^{2}$-gradient flow approach, where the total volume fraction is conserved by adding an extra Lagrange multiplier of nonlocal type. To solve the model, we then develop a semi-discrete in time, easy-to-implement, and second-order scheme. At each time step, one only needs to solve two decoupled linear equations.

Numerical examples show that the added linear stabilization term is extremely important to handle stability for large time step simulation. The unconditional energy stability of the numerical scheme is strictly proved. Compared with other numerical methods in simulating numerous numerical examples, we show the effectiveness and stability 
of the new scheme.

\section{Acknowledgements}

S. Geng was supported by National Natural Science Foundation of China (Nos. 71901150, 71971143), the Natural Science Foundation of Guangdong Province (No. 2020A151501749). Q. Ye was partially supported by Kunming E-Commerce and Internet Finance R\&D Center (KEIRDC[2020]), the Prominent Educator Program (Yunnan[2018]11), Yunnan Province Young Academic and Technical Leader Candidate Program (No. 2018HB027) \& Yunnan Provincial E-Business Entrepreneur Innovation Interactive Space (No. 2017DS012). X. Yang was partially supported by National Science Foundation with grant number DMS-1720212.

\section{References}

[1] A. Aristotelous, O. Karakashian, And S. M. Wise, A mixed discontinuous Galerkin, convex splitting scheme for a modified Cahn-Hilliard equation and an efficient nonlinear multigrid solver, Discrete \& Continuous Dynamical Systems-B, 18 (2013), 2211.

[2] K. BINDER, Collective diffusion, nucleation, and spinodal decomposition in polymer mixtures, J. Chem. Phys., 79 (1983), 6387.

[3] S. A. BRAZOVSKII, Phase transition of an isotropic system to a nonuniform state, JETP, 41(1) (1975), 85.

[4] Q. CHENG AND J. SHEN, Multiple Scalar Auxiliary Variable (MSAV) approach and its application to the phase-field vesicle membrane model, SIAM. J. Sci. Comput., 40 (2018), pp. A3982-A4006.

[5] R. CHOKSI AND X. REN, On the derivation of a density functional theory for microphase separation of diblock copolymers, J. Stat. Phys., 113 (2003), pp. 151-176.

[6] P. G. DE Gennes, Scaling Concepts in Polymer Physics, Cornell University Press, Ithaca, 1979.

[7] P. G. DE GENNES, Dynamics of fluctuations and spinodal decomposition in polymer blends, J. Chem. Phys., 7 (1980), 4756.

[8] D. J. EYRE, Unconditionally gradient stable time marching the Cahn-Hilliard equation, Computational and Mathematical Models of Microstructural evolution (San Francisco, CA, 1998), volume 529 of Mater. Res. Soc. Sympos. Proc., pages 39-46. MRS, Warrendale, PA, 1998.

[9] X. FENG AND A. PROL, Numerical analysis of the allen-cahn equation and approximation for mean curvature flows, Numer. Math., 94 (2003), pp. 33-65.

[10] M. FIALKOWSKI AND R. HOLYST, Dynamics of phase separation in polymer blends revisited: morphology, spinodal, noise, and nucleation, Macromol. Theory Simul., 17 (2008), 263.

[11] G. H. FREDRICKSON, Surface ordering phenomena in block copolymer melts, Macromolecules, 20(6) (1987), pp. 2535-2542.

[12] Y. GAO, X. HE, L. MEI, AND X. YANG, Decoupled, linear, and energy stable finite element method for Cahn-Hilliard-Navier-Stokes-Darcy phase field model, SIAM. J. Sci. Comput., 40 (2018), pp. B110-B137. 
[13] Q. HuANG, X. YANG, AND X. HE, Numerical approximations for a smectic-A Liquid Crystal Flow model: First-order, linear, decoupled and energy stable schemes, Disc. Conti. Dyn. Sys.-B, 23 (2018), pp. 2177-2192.

[14] D. JeOnG, J. Shin, Y. Li, Y. ChOI, J-H. JunG, AND J. Kim S. Lee, Numerical analysis of energy-minimizing wavelengths of equilibrium states for diblock copolymers, Curr. Appl. Phys., 14 (2014), pp. 1263-1272.

[15] D. LeE AND J. KIM, Comparison study of the conservative Allen-Cahn and the Cahn-Hilliard equations, Math. Comput. Simulation, 119 (2016), pp. 35-56.

[16] L. LEIBLER, Theory of microphase separation in block copolymers, Macromolecules, 13(6) (1980), pp. 1602-1617.

[17] T. OHTA AND K. KAWASAKI, Equilibrium morphology of block copolymer melts, Macromolecules, 19(10) (1986), pp. 2621-2632.

[18] J. RUBINSTEIN AND P. STERNBERG, Nonlocal reaction-diffusion equations and nucleation, IMA J. Appl. Math., 48 (1992), pp. 249-264.

[19] J. SHEN, J. XUE, AND J. YANG, The scalar auxiliary variable (sav) approach for gradient flows, J. Comput. Phys., 353 (2018), pp. 407-416.

[20] J. SHEN AND X. YANG, Numerical approximations of Allen-Cahn and Cahn-Hilliard equations, Disc. Conti. Dyn. Sys.-A, 28 (2010), pp. 1669-1691.

[21] J. SHEN AND X. YANG, The IEQ and SAV approaches and their extensions for a class of highly nonlinear gradient flow systems, Contemporary Math., 754 (2020), pp. 217-245.

[22] XIANG-FA WU AND YURIS A DZENIS, Phase-field modeling of the formation of lamellar nanostructures in diblock copolymer thin films under inplanar electric fields, Phys. Rev. E, 77(3) (2008), 031807.

[23] T. Xu, A. V. Zvelindovsky, G. Sevink A, O. GANG, B. OcKO, Y. ZHU, S. P. Gido, AND T. P. RuSSELL, Electric field induced sphere-to-cylinder transition in diblock copolymer thin films, Macromolecules, 37(18) (2004), pp. 6980-6984.

[24] X. YANG, A novel fully-decoupled scheme with second-order time accuracy and unconditional energy stability for the Navier-Stokes equations coupled with mass-conserved Allen-Cahn phase-field model of two-phase incompressible flow, Int. J. Numer. Methods Eng., 122 (2021), pp. 1283-1306.

[25] X. YANG, A novel fully-decoupled, second-order and energy stable numerical scheme of the conserved Allen-Cahn type flow-coupled binary surfactant model, Comput. Methods Appl. Mech. Eng., 373 (2021), 113502.

[26] X. YANG, Numerical approximations of the Navier-Stokes equation coupled with volume-conserved multi-phase-field vesicles system: fully-decoupled, linear, unconditionally energy stable and secondorder time-accurate numerical scheme, Comput. Methods Appl. Mech. Eng., 375 (2021), 113600.

[27] S. ZHAI, Z. WENG, AND X. FENG, Investigations on several numerical methods for the non-local allen-cahn equation, Int. J. Heat Mass Transfer, 87 (2015), pp. 111-118.

[28] J. ZHANG AND X. YANG, Decoupled, non-iterative, and unconditionally energy stable large time stepping method for the three-phase Cahn-Hilliard phase-field model, J. Comput. Phys., 404 (2020), 109115.

[29] J. ZHANG AND X. YANG, Unconditionally energy stable large time stepping method for the L2gradient flow based ternary phase-field model with precise nonlocal volume conservation, Comput. Methods Appl. Mech. Eng., 361 (2020), 112743.

[30] X. ZHANG, J. F. DOUGLAS, AND R. L. JONES, Influence of film casting method on block copolymer ordering in thin films, Soft Matter, 8 (2012), pp. 4980-4986. 